\title{
Pyruvate dehydrogenase deficiency: morphological and metabolic effects, creation of animal model to search for curative treatment
}

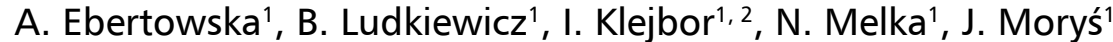 \\ ${ }^{1}$ Department of Anatomy and Neurobiology, Medical University of Gdansk, Poland \\ ${ }^{2}$ Department of Clinical Anatomy, Pomeranian University in Slupsk, Poland
}

[Received: 30 September 2019; Accepted: 3 February 2020]

The main source of energy for brain and other organs is glucose. To obtain energy for all tissue, glucose has to come through glycolysis; then as pyruvate it is converted to acetyl-CoA by pyruvate dehydrogenase complex (PDC) and finally enters citric acid cycle. What happens when one of these stages become disturb? Mutation in genes encoding subunits of PDC leads to pyruvate dehydrogenase deficiency. Abnormalities in PDC activity result in severe metabolic and brain malformations. For better understanding the development and mechanism of pyruvate dehydrogenase deficiency the murine model of this disease has been created. Studies on a murine model showed similar malformation in brain structures as in the patients suffered from pyruvate dehydrogenase deficiency such as reduced neuronal density, heterotopias of grey matter, reduced size of corpus callosum and pyramids. There is still no effective cure for PDC-deficiency. Promising therapy seemed to be ketogenic diet, which substitutes glucose to ketone bodies as a source of energy. Studies have shown that ketogenic diet decreases lactic acidosis and inhibits brain malformations, but not the mortality in early childhood. The newest reports say that phenylbutyrate increases the level of PDC in the brain, because it reduces the level of inactive form of PDH. Experiments on human fibroblast and zebra fish PDC-deficiency model showed that phenylbutyrate is promising cure to PDC-deficiency. This review summarizes the most important findings on the metabolic and morphological effects of PDC-deficiency and research for treatment therapy. (Folia Morphol 2020; 79, 2: 191-197)

Key words: pyruvate dehydrogenase deficiency, metabolic disorders, Leigh syndrome, phenylbutyrate, ketogenic diet

\section{INTRODUCTION}

Glucose is the main source of energy for brain and other organs. To obtain energy for all tissue, glucose has to come through glycolysis; then as pyruvate it is converted to acetyl-CoA by pyruvate dehydrogenase complex (PDC) and finally enters tricarboxylic acid cycle.

Abnormalities in PDC activity results in severe biochemical/cellular, metabolic and morphological/

Address for correspondence: Dr. A. Ebertowska, Department of Anatomy and Neurobiology, Medical University of Gdansk, ul. Dębinki 1, 80-210 Gdańsk, Poland, e-mail: adriana.ebertowska@gumed.edu.pl 
anatomical malformations. Most of the research in this field concerned molecular/biochemical mechanism, but in this review, authors summarise primarily less numerous findings on the metabolic as well as morphological effects of PDC-deficiency and research into effective therapy.

\section{PYRUVATE DEHYDROGENASE COMPLEX}

The PDC plays a key role in converting pyruvate to acetyl-Co. This irreversible oxidative decarboxylation of pyruvate to acetyl-CoA links the glycolytic pathway in the cytosol with the citric acid cycle in the mitochondria [19]. The pyruvate dehydrogenase is a huge complex built of three catalytic enzymes, which collaborate closely with each other. PDC consists of E1 subunit (PDH) - having pyruvate dehydrogenase activity and catalysing pyruvate decarboxylation, E2 subunit - dihydrolipoamide acetyltransferase, which is responsible for acetylation of coenzyme and the E3 subunit - dihydrolipoamide dehydrogenase catalysing the regeneration of oxidised lipoamide form [36]. Furthermore, a PDC complex includes protein $X$, E1-specific kinase and phospo-E1 phosphatase [37].

The $\mathrm{E} 1$ component is a heterotetramer consisting of two subunits $-\alpha$ and $\beta$. Its activity depends on its phosphorylated or dephosphorylated form [28]. The dephosphorylation converts E1 subunit to an active form and is regulated by specific phosphatase, which is one of components of PDC. Consequently, regulation of $\mathrm{E} 1$ subunit impacts the activity of whole complex. The organ where the accumulation of active form of PDC is the highest is the brain [45]. In mammals PDH $\alpha$ component is known in two isoforms, which are encoded in two ways - PDHA1 gene in human's somatic cells (Pdha1 in mouse) is located in a $\mathrm{X}$ chromosome and PDHA2 (Pdha2 in mouse) localised in chromosome 4 and expressed only in testis. The cognizance about $\mathrm{E} 1 \alpha$ genetic record is very useful, because many primary defects occurring in this region are the main reasons a PDC deficiency $[8,29]$.

\section{PYRUVATE DEHYDROGENASE DEFICIENCY AND ITS COROLLARY}

Pyruvate dehydrogenase deficiency is a congenital, metabolic disorder caused by incorrect version of gene encoding respective subunits of PDC. The faulty genes pertain to each of the complex subunits, but the E1 $\alpha$ deficiency is the most frequent [35]. Within mutation of $\mathrm{E} 1 \alpha$ some missense/nonsense as well as deletions and insertions were noticed. Lissens et al. [27] studied a group of 130 mutations. They discovered that insertions and deletions occurred on the exons 10 and 11 the most frequent and the missense/nonsense mutation appeared at all exons. There were also some sex differences, namely they found the missense/nonsense mutation in different exons in male and female. Furthermore, frequency of missense/nonsense mutation was lower in female [27]. Interestingly, the highest amount of mutation in E3 subdivision was observed in Ashkenazi Jews population [2]. There was also known case of missense mutation in gene encoding E2 component of PDC, which resulted in paroxysmal exercise-induced dyskinesia [14].

Glucose is the primary source of energy for every cell in the body. Because the brain is the most energy-demanding organ (brain glucose utilisation accounts for approximately $80 \%$ of whole-body glucose disposal in humans), impaired glucose metabolism results mainly in morphological and hence functional changes of nervous structure. Commonly there were known two forms of pyruvate dehydrogenase disorder symptoms - metabolic (severe neonatal lactic acidosis, hyperlactacidaemia, hyperpurvic acidaemia) and neurological (congenital brain malformations like microcephaly, ventricular enlargement, periventricular leukomalacia, agenesis or absence of pons, corpus callosum, pyramid, likewise neurodevelopmental delay is observed). There were also some characteristic features in appearance noticed $[6,9,21,57]$. Barnerias et al. [3] presented four neurological types comprising (1) malformations in corpus callosum and cortex that occur with early encephalopathy, (2) basal ganglia abnormalities and dysfunction of brainstem, (3) congenital motor disorders with paroxysmal dystonia or neuropathy and (4) chronic axonal neuropathy.

The disease, related with an abnormal activity of pyruvate dehydrogenase is the Leigh syndrome (subacute necrotising encephalomyelopathy). Symptoms of this neurodegenerative congenital disorder were described by the first time in 1951 by Denis Leigh based on case of 7-month-old boy who died due to numerous cerebral abnormalities [26]. The subacute necrotising encephalomyelopathy is the most frequent phenotype of mitochondrial diseases in children, affect in 1:40,000 live births $[15,46]$. Symptoms of this disorder occur in infancy or early childhood (3-12 months, $2^{\text {nd }}$ year of life - the latest) $[43,46]$. Leigh syndrome was shown primarily in a neurological lesion in brain structures like ventricu- 
lomegaly, encephalopathy, changes in the grey matter, changes in basal ganglia; in the research of Lee et al. [25] the putamen was the most lesioned structure among the basal ganglia [13]. There were also pathological changes observed in medulla oblongata and midbrain, particularly in substantia nigra and red nucleus. Lesions in other brain areas like thalamus and cerebellum were also noticed. In the cerebellum atrophy as well in magnetic resonance imaging studies $\mathrm{T} 2$ hyperintensity was observed in hemispheres as well as in vermis.

Therefore, as a conclusion, this is important to mention that among morphological effects of an abnormal activity of pyruvate dehydrogenase a particularly large number applies of brain structures involved in motor function (basal ganglia, cerebellum, red nucleus, pyramids) and cognitive function (cerebral cortex, dentate gyrus, corpus callosum) are involved. Whether this is associated with a higher energy demand for cells in these structures remains an open question and requires further research.

\section{MURINE MODEL OF PDC DEFICIENCY}

The PDC deficiency and its consequences are not completely understood and no fully effective medicine has been discovered. For this reason, scientists from Buffalo University developed murine model of PDC deficiency with deletion of Pdha1 gene. They used Cre-loxP recombination to delete Pdha1 gene encoding alfa subunit of E1 component [17, 22, 31]. The number of offspring born alive was reduced; furthermore, only female pups survived. The null mutation was lethal for male progeny $[41,42]$. Obtained animal model of PDC deficiency showed similar features as cases of human PDC deficiency described in other publication; therefore, it became a promising tool in PDC deficiency studies.

\section{MURINE MODEL OF PDC DEFICIENCY IN RESEARCHES}

Pyruvate dehydrogenase complex is an important factor in a glucose homeostasis and glucose is the main energy substrate for the brain [10]. Therefore, as was mentioned above, the malformations caused by deficiency in PDC occur mostly in a brain. The experiments on a murine model of PDC demonstrated a numerous deformities and abnormalities in brain structures in comparison to control animals. Researches of Buffalo University used the magnetic resonance imaging, spectroscopy studies and apparent diffusion coefficient mapping to evaluate the lesions. The analysis of brain structures in experimental animals showed plenty of morphological and histological changes. Thickness of neocortex was decreased as well as pyriform cortex; density of pyramidal neurons was dramatically reduced. Heterotopias in forebrain particularly in caudate nucleus/putamen, changes in architecture of thalamus-reduced neuronal density in paraventricular, anterodorsal, lateral and medial nuclei, reduced thickness of corpus callosum, reduced size of anterior commissure and lateral olfactory tract and enlarged lateral ventricle were observed. Some structures of forebrain were underdeveloped as well. They also observed disorganisation of neuronal axons in pyramidal decussation, anterior commissure, trigeminal lemniscus, dorsal tegmental decussation, rubrospinal tract and lateral olfactory tract. Some malformations in cerebellum also were observed the most conspicuous feature was the general size of this structure, which was smaller than in control group, also reduced size of cerebellar hemisphere cortex, particularly density of Purkinje and granular neurons as well as cerebellar peduncles. In molecular layer they observed reduced amount of fibres so its general size was decreased. Apparent diffusion coefficient analysis confirms significant cell loss and changes in brain structures. As expected, observed malformations correspond with altered brain tissue observed in the human suffered from PDC deficiency $[41,42]$. The important aspects of PDC deficiency were also biochemical parameters, particularly activity of PDC. Results of this analysis showed that transgenic female mice had wild-type as well as PDC-deficient cells in the brain. The activity of active form and total PDC was reduced by $25 \%$ compared with control female mice. Homogenates of brains from PDC-deficient demonstrated reduced level of $\alpha$ and $\beta$ subunits of PDH in PDC-deficient animals [42].

Transgenic animals were examined on the different stages during development. Previous studies reported that developing brain demands a lot of energy sources right after birth and a PDC level significantly increases in this period $[20,51,54]$. Developmental studies demonstrated that the disturbances in PDC activity impair differentiation and proliferation of neuron cells in both prenatal and postnatal periods. Analysis of brain structures confirmed previous observation like reduction of white matter structures, thickness of neocortex and heterotopias in grey matter [41, 42]. Furthermore, they observed gliosis and significant decrease of neuron number. In a given work were 
behavioural tests performed as well. Obtained results demonstrated that mice with PDC-deficiency suffered from neurological disorders [39].

Brain was not the only structure investigated in mice with PDC-deficiency. Because of important role of PDC in glucose homeostasis and lipid synthesis also liver seemed to be important subject to researches. Choi et al. [7] developed PDC-deficiency mice model with deletion of PDC specific for liver. As a result, they observed reduced body mass in transgenic mice. These studies were based on observation of the carbon movement via PDC and investigated its role in biosynthesis of lipids and generation of energy. The sensitivity of insulin was also examined: the results showed that in the liver and in other tissues the sensitivity of insulin was enhanced. It was suspected that improved sensitivity arises out of the need to keep the normal rates of oxidative phosphorylation, which demand increased usage of free fatty acid [7]. To take a closer look at insulin management in PDC-deficiency one more specific murine model was created. Result showed that acetylo-CoA, which come from pyruvate oxidation due a PDC was very important factor participating in processes involved in insulin secretion from $\beta$-cells [48].

\section{COULD WE FIND A CURE?}

The oldest applied therapy in PDC deficiency was the ketogenic diet. In the early $20^{\text {th }}$ century the ketogenic diet was used as a treatment for epilepsy and uncontrolled seizures but afterward it was superseded by antiepileptic drugs. These days the diet is still in use, but mostly in drug-resistant epilepsy $[1,33,55]$. The aim of ketogenic diet is to substitute the fuel source from carbohydrates to ketone bodies and get effect similar to long-term fasting. In normal condition the energy in human organism is acquired from glucose. During ketogenic diet energy is derived from fatty acid oxidation $[4,18]$. Several studies reported that ketone bodies have neuroprotective effect as they influence to mitochondrial activity improved ATP production and decreased production of free radicals. The influence of ketogenic diet on nervous system is also significant for GABA signalling in neurons as well as supervising the work of neuronal synapses [30, 50, 56]. Ketogenic diet could be also applied in diverse neurological disorders such as Alzheimer disease, Parkinson disease, amyotrophic lateral sclerosis, strokes and other mitochondrial disorders [50]. The abnormality in PDC deficiency arises from incorrect converting pyruvate, which is a product of glycolysis to acetylo-CoA, which is a substrate in tricarboxylic acid cycle [34]. In case of disorders connected with a carbohydrate metabolism, ketogenic diet seems to be a perfect solution to reinstate an energy balance. But what did these results show. Sofou et al. [47] examined a group of 19 PDC-deficient children of different age. Results of ketogenic diet treatment showed positive effect on lactic acidosis, because the level of lactate significantly decreased. In paediatric patients with epileptic seizures reduced frequency or even completely suspension were observed. Improvement was noticed also in neurocognitive functioning as well as motor function. However, the ketogenic diet therapy did not significantly affected life expectancy (median in this study was 6 years) [47]. The beneficial effects like stabilisation and inhibition of brain abnormalities' progression were also observed in other studies $[38,53]$. The effects of ketogenic diet had been also investigated in mice model of PDC-deficiency with the difference that, the special diet was applied to pregnant animals and then during lactation. Therefore, they examined effects of treatment on the progeny. The brain structures in studied progeny (with feeding fat-rich diet) were much better improved than in control group, especially in neocortex, organisation of neurons fibres and cerebellar structures. Furthermore, no structural abnormalities in forebrain and brainstem structures were observed. This study concluded that ketogenic diet during prenatal and early postnatal period was gainful for PDC-deficient mice progeny [40].

The administration of thiamine pyrophosphate (TPP) as an additional treatment can potentially be effective, given its role as an obligate cofactor for the E1 component of the PDC complex. Many cases of PDC deficiency involved a pathological mutation affecting the TPP binding site of E1; supplementation with high doses of thiamine seems justified. The TPP treatment showed improvement in clinical outcomes, such as suspension of ataxia episodes and/or reduced lactate acidosis. These results were confirmed by in vitro studies showing a thiamine-responsive functional defect in the activities of PDC and E1 in the lymphoblastoid cells cultured as well as in muscles and fresh mononuclear cells. Results showed that measurement of TPP is essential in low as well as high activity of PDC to determine the thiamine responsiveness $[3,16,24,44]$. 
Dichloroacetate (DCA) stimulates the activity of the PDC by inhibiting the phosphorylation (and inactivation) of the E1 $\alpha$ subunit of PDC complex. In some patients with $\mathrm{E} 1 \alpha$ deficiency, DCA may also stabilise the enzyme and decrease its rate of turnover.

Several studies had reported that the chronic, oral administration of DCA has been beneficial for patients with PDC deficiency or some patients with respiratory chain defects. DCA treatment was effective in reducing blood, cerebrospinal fluid, or brain lactate concentrations and in improving quality of live. However, cases of peripheral neuropathy and hepatocellular toxicity have been noted $[5,23,32,49]$.

Phenylbutyrate seems to be a promising therapy in PDC-deficiency. This compound is well known and applied in other therapies such as urea cycle disorder, so the activity and effects on healthy tissue are wellknown [52]. Experiments tested in human fibroblasts and zebra model of PDC-deficiency showed that phenylbutyrate increased activity of PDC in brain and other organs. This is because the phenylbutyrate affects the PDC kinase and inhibits it - in consequence the phosphorylated (inactive) form of PDH is reduced, whereas the dephosphorylated form (active) was predominant. Furthermore, the systemic lactic acidosis was reduced [12]. Interestingly, phenylbutyrate in combination with dichloroacetate is much more effective in increasing the activity of PDC in mice. However, the toxicity of these drugs combination is unexplored and demand further tests [11]. The impact of phenylbutyrate on PDC complex activity and its result in brain and other tissue is still examined.

\section{CONCLUSIONS}

Pyruvate dehydrogenase deficiency is a severe metabolic and neurological disorder. Despite many promising therapies applied to patients, none of them is completely effective. Ideally, ketogenic diet should be applied to pregnant mothers and to infants immediately after birth, unfortunately the knowledge about the disorder comes too late. Phenylbutyrate, which reduces the level of inactive form of PDH, is safe for healthy tissue and seems to be hopeful weapon against PDC-deficiency. This therapy, however, demands further examination.

\section{REFERENCES}

1. Acharya MM, Hattiangady B, Shetty AK. Progress in neuroprotective strategies for preventing epilepsy. Prog Neurobiol. 2008; 84(4): 363-404, doi: 10.1016/j.pneurobio.2007.10.010, indexed in Pubmed: 18207302.
2. Ambrus A, Adam-Vizi V. Human dihydrolipoamide dehydrogenase (E3) deficiency: Novel insights into the structural basis and molecular pathomechanism. Neurochem Int. 2018; 117: 5-14, doi: 10.1016/j.neuint.2017.05.018, indexed in Pubmed: 28579060.

3. Barnerias C, Saudubray JM, Touati G, et al. Pyruvate dehydrogenase complex deficiency: four neurological phenotypes with differing pathogenesis. Dev Med Child Neurol. 2010; 52(2): e1-e9, doi: 10.1111/j.14698749.2009.03541.x, indexed in Pubmed: 20002125.

4. Barzegar M, Afghan M, Tarmahi V, et al. Ketogenic diet: overview, types, and possible anti-seizure mechanisms. Nutr Neurosci. 2019 [Epub ahead of print]: 1-10, doi: 10.1080/1028415X.2019.1627769, indexed in Pubmed: 31241011 .

5. Berendzen K, Theriaque DW, Shuster J, et al. Therapeutic potential of dichloroacetate for pyruvate dehydrogenase complex deficiency. Mitochondrion. 2006; 6(3): 126-135, doi: 10.1016/j.mito.2006.04.001, indexed in Pubmed: 16725381.

6. Brown GK, Otero LJ, LeGris M, et al. Pyruvate dehydrogenase deficiency. J Med Genet. 1994; 31(11): 875-879, doi: 10.1136/jmg.31.11.875, indexed in Pubmed: 7853374.

7. Choi CS, Ghoshal P, Srinivasan M, et al. Liver-specific pyruvate dehydrogenase complex deficiency upregulates lipogenesis in adipose tissue and improves peripheral insulin sensitivity. Lipids. 2010; 45(11): 987-995, doi: 10.1007/ s11745-010-3470-8, indexed in Pubmed: 20835892.

8. Dahl HH, Brown RM, Hutchison WM, et al. A testis-specific form of the human pyruvate dehydrogenase $\mathrm{E} 1 \alpha$ subunit is coded for by an intronless gene on chromosome 4 . Genomics. 1990; 8(2): 225-232, doi: 10.1016/08887543(90)90275-y.

9. Dahl HH, Hansen LL, Brown RM, et al. X-linked pyruvate dehydrogenase $\mathrm{E} 1$ alpha subunit deficiency in heterozygous females: variable manifestation of the same mutation. J Inherit Metab Dis. 1992; 15(6): 835-847, doi: 10.1007/ bf01800219, indexed in Pubmed: 1293379.

10. Dienel GA. Brain glucose metabolism: integration of energetics with function. Physiol Rev. 2019; 99(1): 949-1045, doi: 10.1152/physrev.00062.2017, indexed in Pubmed: 30565508.

11. Ferriero R, lannuzzi $C$, Manco G, et al. Differential inhibition of PDKs by phenylbutyrate and enhancement of pyruvate dehydrogenase complex activity by combination with dichloroacetate. J Inherit Metab Dis. 2015; 38(5): 895-904, doi: 10.1007/s10545-014-9808-2, indexed in Pubmed: 25601413.

12. Ferriero R, Manco G, Lamantea E, et al. Phenylbutyrate therapy for pyruvate dehydrogenase complex deficiency and lactic acidosis. Sci Transl Med. 2013; 5(175): 175ra31, doi: 10.1126/scitranslmed.3004986, indexed in Pubmed: 23467562.

13. Finsterer J. Leigh and leigh-like syndrome in children and adults. Pediatr Neurol. 2008; 39(4): 223-235, doi: 10.1016/j. pediatrneurol.2008.07.013, indexed in Pubmed: 18805359.

14. Friedman J, Feigenbaum A, Chuang N, et al. Pyruvate dehydrogenase complex-E2 deficiency causes paroxysmal exercise-induced dyskinesia. Neurology. 2017; 89(22): 
2297-2298, doi: 10.1212/WNL.0000000000004689, indexed in Pubmed: 29093066.

15. Gerards M, Sallevelt SC, Smeets HJM. Leigh syndrome: Resolving the clinical and genetic heterogeneity paves the way for treatment options. Mol Genet Metab. 2016; 117(3): 300-312, doi: 10.1016/j.ymgme.2015.12.004, indexed in Pubmed: 26725255.

16. Giribaldi G, Doria-Lamba L, Biancheri R, et al. Intermittent-relapsing pyruvate dehydrogenase complex deficiency: a case with clinical, biochemical, and neuroradiological reversibility. Dev Med Child Neurol. 2012; 54(5): 472-476, doi: 10.1111/j.1469-8749.2011.04151.x, indexed in Pubmed: 22142326.

17. Gu H, Zou YR, Rajewsky K. Independent control of immunoglobulin switch recombination at individual switch regions evidenced through Cre-loxP-mediated gene targeting. Cell. 1993; 73(6): 1155-1164, doi: 10.1016/00928674(93)90644-6.

18. Gzielo K, Soltys Z, Rajfur Z, et al. The impact of the ketogenic diet on glial cells morphology. A quantitative morphological analysis. Neuroscience. 2019; 413: 239-251, doi: 10.1016/j.neuroscience.2019.06.009, indexed in Pubmed: 31220541.

19. Harris R, Bowker-Kinley $M$, Huang $B$, et al. Regulation of the activity of the pyruvate dehydrogenase complex. Adv Enzyme Regul. 2002; 42: 249-259, doi: 10.1016/s00652571(01)00061-9.

20. Hawkins RA, Williamson DH, Krebs HA. Ketone-body utilization by adult and suckling rat brain in vivo. Biochem J. 1971; 122(1): 13-18, doi: 10.1042/bj1220013, indexed in Pubmed: 5124783.

21. Imbard A, Boutron A, Vequaud C, et al. Molecular characterization of 82 patients with pyruvate dehydrogenase complex deficiency. Structural implications of novel amino acid substitutions in E1 protein. Mol Genet Metab. 2011; 104(4): 507-516, doi: 10.1016/j.ymgme.2011.08.008, indexed in Pubmed: 21914562.

22. Johnson MT, Mahmood S, Hyatt SL, et al. Inactivation of the murine pyruvate dehydrogenase (Pdha1) gene and its effect on early embryonic development. Mol Genet Metab. 2001; 74(3): 293-302, doi: 10.1006/mgme.2001.3249, indexed in Pubmed: 11708858.

23. Kaufmann P, Engelstad K, Wei Y, et al. Dichloroacetate causes toxic neuropathy in MELAS: a randomized, controlled clinical trial. Neurology. 2006; 66(3): 324-330, doi: 10.1212/01. wnl.0000196641.05913.27, indexed in Pubmed: 16476929.

24. Lee EH, Ahn MS, Hwang JS, et al. A Korean female patient with thiamine-responsive pyruvate dehydrogenase complex deficiency due to a novel point mutation (Y161C) in the PDHA1 gene. J Korean Med Sci. 2006; 21(5): 800-804, doi: $10.3346 / \mathrm{jkms} .2006 .21 .5 .800$, indexed in Pubmed: 17043409.

25. Lee HF, Tsai CR, Chi CS, et al. Leigh syndrome: clinical and neuroimaging follow-up. Pediatr Neurol. 2009; 40(2): 88-93, doi: 10.1016/j.pediatrneurol.2008.09.020, indexed in Pubmed: 19135620.

26. Leigh $D$. Subacute necrotizing encephalomyelopathy in an infant. J Neurol Neurosurg Psychiatry. 1951; 14(3): 216-221, doi: 10.1136/jnnp.14.3.216, indexed in Pubmed: 14874135.

27. Lissens W, Meirleir LDe, Seneca S, et al. Mutations in the $X$-linked pyruvate dehydrogenase (E1) ? subunit gene
(PDHA1) in patients with a pyruvate dehydrogenase complex deficiency. Human Mutation. 2000; 15(3): 209-219, doi: 10.1002/(sici)1098-1004(200003)15:3<209::aidhumu1 >3.0.co;2-k.

28. Lissens W, Vreken P, Barth PG, et al. Cerebral palsy and pyruvate dehydrogenase deficiency: identification of two new mutations in the E1alpha gene. Eur J Pediatr. 1999; 158(10): 853-857, doi: 10.1007/s004310051222, indexed in Pubmed: 10486093.

29. Maragos C, Hutchison WM, Hayasaka K, et al. Structural organization of the gene for the $\mathrm{E} 1$ alpha subunit of the human pyruvate dehydrogenase complex. J Biol Chem. 1989; 264(21): 12294-12298, indexed in Pubmed: 2745444.

30. Masino SA, Rho JM. Mechanisms of Ketogenic Diet Action. National Center for Biotechnology Information. (US): 2012.

31. Nagy A. Cre recombinase: The universal reagent for genome tailoring. Genesis. 2000; 26(2): 99-109, doi: 10.1002/(sici)1526-968x(200002)26:2<99::aidgene1>3.0.co;2-b.

32. Naito E, Kuroda Y, Takeda E, et al. Detection of pyruvate metabolism disorders by culture of skin fibroblasts with dichloroacetate. Pediatr Res. 1988; 23(6): 561-564, doi: 10.1203/00006450-198806000-00005, indexed in Pubmed: 2839811.

33. Neal E, Chaffe H, Schwartz R, et al. The ketogenic diet for the treatment of childhood epilepsy: a randomised controlled trial. Lancet Neurol. 2008; 7(6): 500-506, doi: 10.1016/s1474-4422(08)70092-9.

34. Ng F, Tang BL. Pyruvate dehydrogenase complex (PDC) export from the mitochondrial matrix. Mol Membr Biol. 2014; 31(7-8): 207-210, doi: 10.3109/09687688.2014.9 87183, indexed in Pubmed: 25495576.

35. Patel KP, O'Brien TW, Subramony SH, et al. The spectrum of pyruvate dehydrogenase complex deficiency: clinical, biochemical and genetic features in 371 patients. Mol Genet Metab. 2012; 105(1): 34-43, doi: 10.1016/j. ymgme.2011.09.032, indexed in Pubmed: 22079328.

36. Patel MS, Nemeria NS, Furey W, et al. The pyruvate dehydrogenase complexes: structure-based function and regulation. J Biol Chem. 2014; 289(24): 16615-16623, doi: 10.1074/jbc.R114.563148, indexed in Pubmed: 24798336

37. Patel MS, Roche TE. Molecular biology and biochemistry of pyruvate dehydrogenase complexes. FASEB J. 1990; 4(14): 3224-3233, doi: 10.1096/fasebj.4.14.2227213, indexed in Pubmed: 2227213.

38. Di Pisa V, Cecconi I, Gentile V, et al. Case report of pyruvate dehydrogenase deficiency with unusual increase of fats during ketogenic diet treatment. J Child Neurol. 2012; 27(12): 1593-1596, doi: 10.1177/0883073812436424, indexed in Pubmed: 22378660.

39. Pliss L, Hausknecht KA, Stachowiak MK, et al. Cerebral developmental abnormalities in a mouse with systemic pyruvate dehydrogenase deficiency. PLoS One. 2013; 8(6): e67473, doi: 10.1371/journal.pone.0067473, indexed in Pubmed: 23840713.

40. Pliss L, Jatania $U$, Patel MS. Beneficial effect of feeding a ketogenic diet to mothers on brain development in their progeny with a murine model of pyruvate dehydro- 
genase complex deficiency. Mol Genet Metab Rep. 2016; 7: 78-86, doi: 10.1016/j.ymgmr.2016.03.012, indexed in Pubmed: 27331005.

41. Pliss L, Mazurchuk R, Spernyak JA, et al. Brain MR imaging and proton MR spectroscopy in female mice with pyruvate dehydrogenase complex deficiency. Neurochem Res. 2007; 32(4-5): 645-654, doi: 10.1007/s11064-007-9295-z, indexed in Pubmed: 17342409.

42. Pliss L, Pentney RJ, Johnson MT, et al. Biochemical and structural brain alterations in female mice with cerebral pyruvate dehydrogenase deficiency. J Neurochem. 2004; 91(5): 1082-1091, doi: 10.1111/j.14714159.2004.02790.x, indexed in Pubmed: 15569252.

43. Rahman S, Blok RB, Dahl HH, et al. Leigh syndrome: clinical features and biochemical and DNA abnormalities. Ann Neurol. 1996; 39(3): 343-351, doi: 10.1002/ ana.410390311, indexed in Pubmed: 8602753.

44. Di Rocco M, Lamba LD, Minniti G, et al. Outcome of thiamine treatment in a child with Leigh disease due to thiamine-responsive pyruvate dehydrogenase deficiency. Eur J Paediatr Neurol. 2000; 4(3): 115-117, doi: 10.1053/ ejpn.2000.0278, indexed in Pubmed: 10872106.

45. Siess E, Wittmann J, Wieland O. Interconversion and kinetic properties of pyruvate dehydrogenase from brain. Hoppe Seylers Z Physiol Chem. 1971; 352(3): 447-452, doi: 10.1515/ bchm2.1971.352.1.447, indexed in Pubmed: 5550962.

46. Sofou K, De Coo IFM, Isohanni P, et al. A multicenter study on Leigh syndrome: disease course and predictors of survival. Orphanet J Rare Dis. 2014; 9: 52, doi: 10.1186/17501172-9-52, indexed in Pubmed: 24731534.

47. Sofou K, Dahlin M, Hallböök T, et al. Ketogenic diet in pyruvate dehydrogenase complex deficiency: short- and long-term outcomes. J Inherit Metab Dis. 2017; 40(2): 237-245, doi: 10.1007/s10545-016-0011-5, indexed in Pubmed: 28101805.

48. Srinivasan M, Choi CS, Ghoshal P, et al. B-Cell-specific pyruvate dehydrogenase deficiency impairs glucose-stimulated insulin secretion. Am J Physiol Endocrinol Metab. 2010; 299(6): E910-E917, doi: 10.1152/ajpendo.00339.2010, indexed in Pubmed: 20841503.
49. Stacpoole PW, Kerr DS, Barnes C, et al. Controlled clinical trial of dichloroacetate for treatment of congenital lactic acidosis in children. Pediatrics. 2006; 117(5): 1519-1531, doi: 10.1542/peds.2005-1226, indexed in Pubmed: 16651305.

50. Stafstrom C, Rho J. The Ketogenic Diet as a Treatment Paradigm for Diverse Neurological Disorders. Front Pharmacol. 2012; 3: 59, doi: 10.3389/fphar.2012.00059.

51. Takakubo F, Dahl HH. Analysis of pyruvate dehydrogenase expression in embryonic mouse brain: localization and developmental regulation. Dev Brain Res. 1994; 77(1): 63-76, doi: 10.1016/0165-3806(94)90214-3.

52. Tuchman M, Lee B, Lichter-Konecki U, et al. Cross-sectional multicenter study of patients with urea cycle disorders in the United States. Mol Genet Metab. 2008; 94(4): 397-402, doi: 10.1016/j.ymgme.2008.05.004, indexed in Pubmed: 18562231.

53. Wijburg FA, Barth PG, Bindoff LA, et al. Leigh syndrome associated with a deficiency of the pyruvate dehydrogenase complex: results of treatment with a ketogenic diet. Neuropediatrics. 1992; 23(3): 147-152, doi: 10.1055/s2008-1071331, indexed in Pubmed: 1641082.

54. Wilbur DO, Patel MS. Development of mitochondrial pyruvate metabolism in rat brain. J Neurochem. 1974; 22(5): 709-715, doi: 10.1111/j.1471-4159.1974.tb04284.x, indexed in Pubmed: 4407094.

55. Williams TJ, Cervenka MC. The role for ketogenic diets in epilepsy and status epilepticus in adults. Clin Neurophysiol Pract. 2017; 2: 154-160, doi: 10.1016/j.cnp.2017.06.001, indexed in Pubmed: 30214989.

56. Włodarek D. Role of ketogenic diets in neurodegenerative diseases (Alzheimer's disease and Parkinson's disease). Nutrients. 2019; 11(1), doi: 10.3390/nu11010169, indexed in Pubmed: 30650523.

57. Yoshida T, Kido J, Mitsubuchi H, et al. Clinical manifestations in two patients with pyruvate dehydrogenase deficiency and long-term survival. Hum Genome Var. 2017; 4: 17020, doi: 10.1038/hgv.2017.20, indexed in Pubmed: 28584645. 